\title{
Determinación de la asociación entre Chlamydia trachomatis y Neisseria gonorrhoeae, en muestras de orina mediante técnicas de biología molecular
}

\author{
${ }^{1}$ Gabriela Coy Bsc, ${ }^{1}$ Olga Lucía Ostos Msc, ${ }^{2}$ Janeth Pinzón Bsc, ${ }^{1}$ Ruth Mélida Sánchez Msc. \\ ${ }^{1}$ Facultad de Ciencias de la Salud, Universidad Colegio Mayor de Cundinamarca, ${ }^{2}$ Laboratorio de Biología Molecular de la \\ Secretaria de Salud de Bogotá. \\ Recibido:23-07-2004; Aceptado: 13-10-2004
}

\begin{abstract}
Resumen
En el presente estudio se evaluó la asociación entre Chlamydia trachomatis (CT) y Neisseriae gonorrhoeae (NG), utilizando la técnica de COBAS AMPLICOR CT/NG Molecular System, en muestras de orina de 355 mujeres con un promedio de edad de 23.9 años; 20 muestras fueron positivas para CT, informándose una prevalencia de de 5.63\%. Aunque estudios epidemiológicos han reportado relación entre la infección por Chlamydia trachomatis y las causadas por otros microorganismos como Neisseriae gonorrhoeae; en este estudio no se encontró asociación. Factores como el tipo de muestra y la presencia de inhibidores en la orina disminuyen la sensibilidad en la detección de Neisseriae gonorrhoeae.

Palabras claves: Chlamydia trachomatis, asociación, Neisseriae gonorrhoeae, PCR, sensibilidad, orina.

\section{Abstract \\ Determine the association Between Chlamydia Trachomatis and Neisseriae Gonorrhoeae through molecular biology techniques in urine samples.}

The following study evaluated the association between Chlamydia Trachomatis (CT) and Neisseriae Gonorrhoeae (NG) using COBAS AMPLICOR CT/NG MOLECULAR SYSTEM technique in 35 women urine samples with a 23.9 year old average. 20 samples were positive to $\mathrm{CT}$, showing a prevalence of $5.63 \%$. Although, epidemiological studies have reported a relationship between Chlamydia Trachomatis infection and the ones caused by others microorganisms like Neisseriae Gonorrhoeae; this study didn't find any association. Factors like kind of sample and the presence of inhibitors in urine, diminished sensibility to detect Neisseriae Gonorrhoeae.
\end{abstract}

Key Words: Chlamydia Trachomatis, association, Neisseriae Gonorrhoeae, PCR, sensibility, urine.

\section{Introducción}

El patógeno humano Neisseria gonorrhoeae $(\mathrm{NG})$ es un coco gram negativo, no móvil, no esporulado, el cual crece característicamente en pares (diplococo),

Correspondencia: olostos@unicolmayor.edu.co es susceptible a los cambios ambientales y no sobrevive por largos periodos fuera de su huésped; considerándose un parásito obligado del hombre. La infección causada por el patógeno es conocida como infección gonococcica; el microorganismo crece sobre 
las células epiteliales y es capaz de invadir las barreras y diseminarse vía sanguínea a múltiples órganos incluyendo el fluido cerebro espinal, piel y articulaciones (1). La unión a la mucosa esta mediada por pilis o proteínas, veinticuatro a cuarenta y ocho horas después ocurre una penetración del microorganismo entre y a través de las células epiteliales a los tejidos de la submucosa, lo que genera una respuesta inflamatoria mediada por neutrófilos, con necrosis del epitelio $(1,2)$.

Por otra parte, Chlamydia trachomatis (CT), es una bacteria intracelular obligada, presenta una morfología esférica u ovalada y se observan como cocos gram negativos o gram variables, son inmóviles no ciliadas, con una membrana interna y otra externa y tienen un ciclo de crecimiento único que implica dos formas denominadas cuerpo elemental y cuerpo reticulado o inicial, estos representan las formas extracelulares e intracelulares del parásito, respectivamente.

Las complicaciones clínicas en mujeres causadas por CT son; absceso pélvico, enfermedad pélvica inflamatoria crónica y recurrente, embarazo ectópico e infertilidad; en hombres causa infección genital crónica del tracto y en niños conjuntivitis y neumonía (3).

Estudios epidemiológicos, han encontrado evidencias de una relación entre la infección por CT y las causadas por otros microorganismos como por NG (4$8)$, tal vez originado por el debilitamiento de las barreras fisiológicas y de los mecanismos inmunológicos de defensa del organismo, que permite una mayor susceptibilidad a la invasión por estos patógenos $(4,5)$. De acuerdo a lo anterior, nos preguntamos, sí ¿es posible detectar en muestra de orina de estos grupos de pacientes la asociación entre CT y NG?

El estudio evaluó dicha asociación, utilizando la técnica de COBAS AMPLICOR/NG Molecular System, en 355 muestras de orina de mujeres con un promedio de edad de 23.9 años.

www.unicolmayor.edu.co

\section{Materiales y métodos}

Población accesible y muestra: la población accesible fue de 4500 mujeres menores de treinta años con vaginitis inespecífica, que asistían a los hospitales de la Secretaria de Salud de Bogotá y una población de igual tamaño de mujeres jóvenes asintomáticas. Mediante el análisis de potencia se determinó una muestra de 175 mujeres jóvenes con vida sexual activa y diagnóstico clínico de vaginitis inespecífica y 168 mujeres jóvenes asintomáticas con vida sexual activa, se utilizó un diseño muestral aleatorio estratificado.

Obtención de la muestra: se analizaron 10 a 50ml de orina, obtenidos de la primera micción del día (flujo inicial del chorro), recolectados en frascos limpios de polipropileno. Para determinar el tipo de riesgo de la población de contraer alguna enfermedad de transmisión sexual, se realizó una encuesta siguiendo las recomendaciones del tratado de Helsinki, los consentimientos informados fueron obtenidos de todas las participantes en este estudio.

Preparación de la muestra: la muestra de orina fué homogenizada y tratada con una solución detergente para liberar el $A D N$, y posteriormente se le adicionó una solución diluyente para preparar el espécimen para la amplificación.

Amplificación por PCR: en la amplificación se utilizaron los primers biotinilados específicos, para amplificar una secuencia de aproximadamente 207 pares de bases. Una vez realizada la extracción, el ADN con la mezcla maestra (primers, dNTPs, Taq polimerasa, cloruro de magnesio y buffer) se llevó al termociclador del analizador COBAS AMPLICOR, en donde se realizó la técnica de PCR y posterior detección. Para cada muestra se utilizó un control de amplificación interno.

Reacción de hibridación: una vez realizada la $P C R$, se realizó la reacción de hibridación desnaturalizando los amplicones, estos se colocaron en los pocillos de detección que contenían las partículas magnéticas revestidas con una sonda 
oligonucleotida especifica para NG. Los amplicones marcados con botina hibridan con las sondas conjugadas con las partículas magnéticas.

Reacción de detección: la detección se realizó en el analizador COBAS AMPLICOR que extrae el conjugado no ligado, lava las partículas magnéticas, y añade un conjugado de avidina- peroxidasa de rábano picante, este último se conjuga con el amplicon marcado con biotina e hibrida con la sonda fijada a las partículas magnéticas, finalmente, se añadió una solución de peróxido de hidrogeno y 3-3', 5-5, tetrametilbencidina (TMB) a cada pozo.

\section{Resultados}

En el presente estudio se evaluó la asociación entre CT y NG, utilizando la técnica de COBAS AMPLICOR CT/NG Molecular System, en muestras de orina de 355 mujeres con un promedio de edad de 23.9 años, donde el 49,3\% fueron mujeres asintomáticas que manifestaron encontrarse totalmente libres de síntomas relacionados con infección vaginal o uretral y el 50,7\% manifestaron presentar sintomatología de vaginitis inespecífica. El promedio de edad de las mujeres participantes fue de 23.9 años (Tabla 1).

Veinte muestras fueron positivas para CT, informándose una prevalencia de $5.63 \%$; esta misma población se utilizó para detectar NG, mediante COBAS AMPLICOR NG Molecular System.
En relación con los antecedentes de las enfermedades vaginales, en el grupo de mujeres asintomáticas, el 79\% manifiesto nunca haber presentado síntomas; en la población sintomática positiva para $\mathrm{CT}$, se encontró que el $33.3 \%$ había padeció enfermedad pélvica inflamatoria anteriormente, de igual forma un 33.3\%, manifestó no saber que enfermedad habían padecido. Las participantes de los dos grupos indicaron en un $24 \%$ haber tenido relaciones sexuales con alguien más aparte de su pareja durante los últimos 6 meses.

El comportamiento sexual de las mujeres que participaron en el estudio se resume en la Tabla 2.

Al realizar la detección de NG en las muestras estudiadas, el $100 \%$ presentó un resultado negativo, lo que indicó un $0 \%$ de prevalencia de Infección gonocócica en la población de estudio. Las muestras positivas para $\mathrm{CT}$, también fueron negativas para $\mathrm{NG}$, es decir, no se encontró asociación entre positividad para CT e infección por NG, mediante PCR CT/NG COBAS AMPLICOR.

\section{Discusión}

En el estudio no se encontró asociación entre la presencia de CT y la detección de NG en las 355 mujeres (promedio de edad de 23.9 años) evaluadas. De las 20 muestras positivas para $\mathrm{CT}$, ninguna fue positiva para $\mathrm{NG}$, el resultado anterior puede estar influenciado por el tipo de muestra utilizada, debido a que el gonococo tiene preferencia para infectar e invadir la uretra, el recto, la

Tabla 1. Distribución de mujeres que participaron en el estudio por estratos que corresponden a su condición de asintomáticas o que presentaban vaginitis inespecífica, se muestra promedio de edades por estrato y porcentaje de positivas para CT y NG

\begin{tabular}{|c|c|c|c|c|c|}
\hline Estratos & $\begin{array}{c}\text { Número de } \\
\text { muestras }\end{array}$ & $\begin{array}{c}\text { Promedio } \\
\text { de edad }\end{array}$ & $\begin{array}{c}\text { Positivas } \\
\text { CT }\end{array}$ & Porcentaje \% & $\begin{array}{c}\text { Positivas } \\
\text { NG }\end{array}$ \\
\hline Mujeres asintomáticas & 175 & 23.3 & 6 & 3.43 & 0 \\
\hline $\begin{array}{c}\text { Mujeres con diagnóstico } \\
\text { de vaginosis y vaginitis } \\
\text { inespecifica }\end{array}$ & 180 & 24.5 & 14 & 7.78 & 0 \\
\hline Total & 355 & 23.9 & 20 & 5.63 & 0 \\
\hline
\end{tabular}


Tabla 2. Características de las mujeres que participaron en el estudio, teniendo en cuenta variables de interés en infección por CT y NG; número de parejas sexuales, números de abortos y uso de preservativo, se muestra la relación de los hallazgos con los resultados positivos de la prueba para CT y NG. NR*; mujeres positivas que no responden la encuesta.

\begin{tabular}{|c|c|c|c|c|c|c|}
\hline Caracteristicas & Asintomáticas & $\begin{array}{c}\text { Positivas } \\
\text { CT }\end{array}$ & $\begin{array}{c}\text { Positivas } \\
\text { NG }\end{array}$ & Sintomáticas & $\begin{array}{c}\text { Positivas } \\
\text { CT }\end{array}$ & $\begin{array}{l}\text { Positivas } \\
\text { NG }\end{array}$ \\
\hline \multicolumn{7}{|l|}{$\begin{array}{c}\text { Número parejas } \\
\text { sexuales }\end{array}$} \\
\hline 1 & 111 & 0 & 0 & 164 & 3 & 0 \\
\hline 2 & 45 & 0 & 0 & 10 & 6 & 0 \\
\hline 30 más & 12 & 6 & 0 & 3 & 5 & 0 \\
\hline $\mathrm{NR}^{*}$ & 7 & 1 & 0 & 0 & 0 & 0 \\
\hline \multicolumn{7}{|l|}{ Número de abortos } \\
\hline ningìno & 149 & 2 & 0 & 146 & 12 & 0 \\
\hline 1 & 18 & 4 & 0 & 27 & 2 & 0 \\
\hline 20 mas & 8 & 0 & 0 & 7 & 0 & 0 \\
\hline \multicolumn{7}{|l|}{ Uso de preservativo } \\
\hline Si & 92 & 2 & 0 & 35 & 1 & 0 \\
\hline No & 83 & 4 & 0 & 145 & 13 & 0 \\
\hline
\end{tabular}

conjuntiva, la faringe y el endocervix, donde los pilis y la proteína II median parcialmente la fijación de este microorganismo a las células mucosas de estos tejidos. Sin embargo, la muestra de orina presenta la ventaja de no ser invasiva, lo que facilita su recolección en hombres y mujeres (6-8); así mismo, se han observado buenos resultados al evaluar un gran número de individuos en estudios de prevalencia del patógeno $(9,10)$ y existen técnicas que pueden ser usadas para la detección simultánea de patógenos en una sola muestra $(10,11)$, así, los sistemas de amplificación para la detección simultánea de infecciones gonocócicas y por Chlamydia, muestran una sensibilidad superior comparados con el método tradicional de cultivo y permite el uso de muestras no invasivas. Lo anterior, incrementa el alcance, disminuye los costos de la salud pública en los programas de tamizaje con el objeto de controlar estas infecciones y permite que instituciones con escasos recursos, desarrollen programas de promoción y prevención en enfermedades de transmisión sexual $(11,12,13)$. Así mismo, diferentes estudios, han comparado la sensibilidad de las técnicas moleculares para la detección de CT y NG en muestras como: hisopado vaginal y muestras de orina, encontrando una alta similitud (90\%) en los resultados obtenidos (14-16).
La ausencia de infección por NG en las muestras analizadas, puede indicar una baja prevalencia de este patógeno en la muestra estudiada. Estudios en otras poblaciones han reportado baja prevalencia de la infección gonococcica, tal es el caso del estudio realizado en noviembre de 2002, en el Hospital General de Montreal en Canadá (17), en el que a 9772 pacientes hombres y mujeres, se les detecto NG utilizando muestras endocervicales, uretrales y de orina, los resultados de este estudio indicaron una baja prevalencia $(0.5 \%)$ y confirmaron la alta sensibilidad $(100 \%)$ y especificidad (98.3-99.2\%) de la técnica COBAS AMPLICOR CT/NG en la detección de $\mathrm{NG}$, el valor predictivo positivo fue de $28,3 \%$ para muestras de orina de mujeres y $7,1 \%$ en muestras endocervicales (17). Sin embargo, estudios realizados por Barbara Van Der Pol, et al. (18), en Indiana (2001), reportan que la técnica COBAS AMPLICOR CT/NG presenta reacción cruzada con algunos aislamientos de Neisseria subflava y Neisseria cinerea, y otros han mostrado que poblaciones con baja prevalencia de CT y NG tienen un valor predictivo positivo bajo, secundario a la reactividad cruzada que se presenta con algunas cepas de Neisseria no patógenas o de Lactobacillus en muestras endocervicales $(18,19)$. 
A pesar de las desventajas informadas del uso de técnicas de biología molecular en algunas poblaciones a nivel mundial, estas continúan siendo una buena alternativa en el diagnóstico de patógenos causantes de enfermedades infecciosas(20) que pueden cursar de forma asintomática o con manifestaciones muy leves, como es el caso de NG, en el que la lectura en mujeres de frotis directos para su diagnóstico no es concluyente (21-23), por lo que se hace necesario realizar cultivos que generalmente son negativos $(24,25)$.

Como recomendación para mejorar la sensibilidad y especificidad en el diagnóstico de NG mediante la técnica COBAS AMPLICOR $\mathrm{CT} / \mathrm{NG}$, proponemos utilizar una muestra de hisopado vaginal disuelto en orina, que permite evaluar en una sola muestra la presencia de CT y NG en patologías como cervicitis y/o uretritis, disminuyendo los costos y mejorando la calidad del diagnóstico.

\section{Referencias}

1. Todar K. The pathogenic Neisseria. University of WisconsinMadison Department of Bacteriology; 2002.

2. Merz AJ. Interactions of pathogenic Neisseria with epithelial cell membranes. Annu rev cell dev biol 2000; (16): 423-57.

3. Kanoh Y. The infections by Chlamydia trachomatis and Neisseria gonorrhoeae Rinsho Byori 2002; 123 (Suppl Nov):57-61.

4. Wiesenfeld HC. Bacterial vaginosis is a strong predictor of Chlamydia trachomatis and Neisseria gonorrhoeae infection. Clin Infecto Dis 2003; 36(5): 663-8.

5. Knox J. Evaluation of self-colected samples in contrast to practitioner-collected samples for detection of Chlamydia trachomatis, Neisseria gonorrhoeae and Trichomonas vaginalis $\mathrm{PCR}$ among Women living in remote areas. Sex transm dis 2002; 29(11): 647

6. Koumans EH. Comparison of methodos for detection of Chlamydia trachomatis and Neisseria gonorrhoeae using commercially available nucleic acid amplification test and liquid pap smear medium. Journal of clinical microbiology 2003; 41(4):1507-11.

7. Bachmann LH, Prevalence and factors associated with gonorrhoea and chamydial infection in at-risk females presenting to an urban emergency department. Sex Transm dis 2003 ; 30(4): 335-9.

8. Castillo MC. Purification and characterization of lactamase from Neisseria gonorrhoeae from clinical samples Chlamydia trachomatis and Neisseria gonorrhoeae in urine specimens, female endocervical swabs and male urethral swabs. Revista latinoamericana de microbiologia 2001; 43(2):70-5.

9. Birch NC. Evaluation of urine specimen integrity in a public health STD screening program. Am J Pathol 2003; 119(4):516-21.

10. Ministerio de salud pública. Informe epidemiológico Nacional INS: 2001; Nov 30; 6(22).

11. Carroll K. Evaluation of the ABBOTT LCx ligase chain reaction assay for detection of Chlamydia trachomatics and Neisseria gonorrhoeae in urine and genital swab specimens from a sexually transmitted diseases clinic population. Journal of clinical microbiology 1998; 36(6):1630-3.

12. Farrell D. Evaluation of AMPLICOR Neisseria gonorrhoeae PCR using cppB nested PCR and 16S rRNA PCR. Journal of clinical microbiology 1999; 37(2): 386-90

13. Hardick J. Surveillance of Chlamydia trachomatis and Neisseria gonorrhoeae infections in women in detection in Baltimore, Maryland. Sex Transm dis 2003; 30(1): 64-70.

14. Taffa N. Prevalence of gonococcal and chlamydial infections and sexual risk behaviour among youth in Addis Adaba, Ethiopia. Sex transm dis 2002; 29(12): 828-33.

15. Palmer HM. Evaluation of the specificities of five DNA amplification methods for the detection of Neisseria gonorrhoeae Journal of Clinical Mycrobiology 2003; 41(2): $835-7$.

16. Schachter J. Which test is best for Chlamydia? Curr Opin Infect Dis 1999; 12;41-5.

17. Van der pol B, et al. Multicenter evaluation of the BD ProbeTec ET system for detection of Chlamydia trachomatis and Neisseria gonorrhoeae in urine specimens, female endocervical swabs and male urethral swabs. Journal of clinical microbiology 2001; 39(3): 1008-16.

18. Newman SB, et al. Female prisioners preferences of collection methods for testing for Chlamydia trachomatis and Neisseria gonorrhoeae infection. Sex Transm dis 2003; 30(4): 306-9.

19. Van doornum GJ. Comparison between the LCx probe system and the COBAS AMPLICOR system for detection of Chlamydia trachomatis and Neisseria gonorrhoeae. Infections in patients attending a clinic for treatment of sexually transmitted diseases in amsterdam the Netherlands. Journal of clinical microbiology 2001; 39(3): 829-35.

20. Makepeace B, et al. Interactions of Neisseria gonorrhoeae with Mature Human Macrophage Opacity Proteins Influence Production of Proinflammatory Cytokines. Infection and immunity 2001; 69(3): 1909-13.

21. Massari P. The role of porins in neisserial pathogenesis and immunity. Trends Microbiol 2003; 11(2):87-93.

22. Mazzone $\mathrm{M}$, et al. Whole-genome organization and functional properties of miniature DNA insertion sequences conserved in pathogenic Neisseria. Gene 2001; 278(1-2): 211-22.

23. Mcknew DL, et al. Porin variation among clinical isolates of Neisseria gonorrhoeae over a 10 year period, as determinated by variable region typing. $J$ Infection disease $2003 ; 187(8)$ : 1213-22

24. Rahman M, et al. Etiology of sexually transmitted infections among street based female sex workers in Dhaka Bangladesh. Journal of microbiology 2000; 38(3): 1244-6.

25. Pujol C, et al. Do pathogenic Neisseria need several ways to modify the host cell cytoskeleton? Microbes and infection $2000 ; 2(7) 821-7$ 